Although it is far beyond the scope of this brief commentary to present a comprehensive view of what may be called a proper account of the meaning of 'subjective', it must be mentioned that Descartes' distinction, though frequently attacked, still underlies most of our concepts and differentiations relevant to the point in question. The difference between 'signs' and 'symptoms', for example, is based on the fact that there is a large difference between my pain and anybody else's pain, as well as between pain and pain behaviour. Contrary to what some Wittgensteinian philosophers want to make us believe, we all have an intuitive grasp that our sensations and thoughts are accessible to us in a different manner than they are to somebody else. In fact, the clinician - whether practising internal medicine or psychiatry - is particularly aware of this difference. Every clinician struggles against this 'epistemological barrier' when trying to obtain a clear picture of any particular symptom.

Just as pain remains a (subjective) symptom when rated on a scale by the patient or by the observer, hallucinations and delusions remain (subjective) symptoms. Moreover, talking about pain or hallucinations does not make the pain or hallucinations intersubjectively more accessible. Their presence has merely been communicated. For example, why are we suspicious of studies claiming to have successfully 'treated' patients with auditory hallucinations by negative reinforcement using self-administered electric shocks? Obviously, because we distinguish not reporting hallucinations from not experiencing them. In short, neither rating a symptom, nor talking about a symptom, 'objectifies' it.

In our view, the acceptance and proper appreciation of the patient as experiencing subject is important to psychiatry and to medicine in general. The view that 'subjective' means 'atmospheric' or 'not delineated' does not help to achieve this goal. Nor does the relating of 'diffuse' and 'subjective' help in getting rid of the diffuseness. Only a clarified concept of the subjective as the realm of what is actually experienced by the patient, in Dr van Praag's words, will help to "expand and refine diagnostic concepts and corresponding psychometric instruments" (p. 270). Without a clear view of the subjective, "we end up with a severely coarsened psychiatry obsessed with the obvious [and] detached from the experiential realm" (p.270).

Klinikum der Universitat Heidelberg

MANFREd SPITZER

Psychiatrische Klinik

Voßstraße 4

6900 Heidelberg

Germany

New York
AUTHOR'S REPLY: My remarks on subjective psychopathological phenomena were pulled out of context by my critics. I reasoned that the prevailing psychiatric classification system based on the DSM-III caters to an overly objective approach, neglecting the realm of the subjective experiences. In that context I described subjective phenomena as those that are confined to the patient's experiential world, not expressed in observable behaviour, and 'atmospheric' rather than 'factual' in nature, that is, not manifesting themselves as delineated mental phenomena and not verbalised as such. Hence, they are largely disregarded in the present psychiatric taxonomy. I did not state that the degree of 'delineation' of mental phenomena is a criterion for their degree of subjectivity, nor did I infer such opinion in my exposition on quasi-subjective symptoms.

A second group of psychopathological phenomena I called subjective are those that are not communicated by the patient as such, but are inferences made by the observer/interviewer - "concepts" construed by incongruous observations. As an example I mentioned the meaning of a particular type of behaviour or utterance. I did not state that theoretically driven constructs are per definition subjective.

My paper did not, by any means, pretend to give a definitive description of the term 'subjective', yet my definition of what is nowadays called 'subjective' in (research) psychiatry seems to me accurate. The dissertation of Drs Spitzer and Schwartz does not provide evidence to the contrary.

Herman M. van Praag

Montefiore Medical Center

Department of Psychiatry

111 210th Street

Bronx

New York 10467

USA

\section{Guilt or morbid remorse?}

SIR: In their interesting discussion of the phenomenology of psychological guilt (Journal, June 1992, 160, 781-787), Berrios et al appear to use the word 'remorse' either as a defining component of the phenomenon or as a synonym. I would submit that these words have a different meaning; 'guilt' implying a consciousness of having committed a wrong, and 'remorse' implying an emotion of regret for the consequences of the wrongful act. I would further submit that the term 'morbid remorse' is a more accurate description of what is encountered in major depression. 
Just as with morbid jealousy, with which it has many parallels, morbid remorse can have a delusional, or real but overvalued, precipitating cause, or may also not have any cause which the patient is able to articulate. For example, it is not uncommon for a depressed elderly patient to feel that they have ruined the lives of their children. Their preoccupation and distress is with the consequence of their imagined short-comings and is therefore better described as a morbid remorse, than as a sense of guilt for being an imperfect parent, a guilt which probably all parents would acknowledge. To say that they are "filled with remorse" conveys a more empathic understanding of their mental state than to suggest that they are "experiencing feelings of guilt".

Regional Treatment Centre (O)

G. N. CONACHER

P.O. Box 22

Kingston, Ontario

K7L 4V7

Availability of the Hospital Anxiety and Depression (HAD) Scale

SIR: In recent issues, Rice \& Donnelly (Psychiatric Bulletin, June 1992, 16, 329-330) found that the HAD Scale was used in clinical work by consultant psychiatrists, and Tyrer (Journal, June 1992, 160, 733-741) recommended its use in the assessment of pain. I welcome the opportunity to inform readers of its present availability. Soon after publication of the study introducing the HAD it was made freely available by the good offices of the Medical Liaison Service of Upjohn. After some years that service had to cease. The HAD is now produced in convenient single-sheet format with inbuilt scoring device, together with a chart for successive recording of scores in order to indicate progress under treatment. For bulk supply of this material a charge is necessary to cover costs; alternatively, single copies may be obtained and personal copies made by the user.

Despite the word 'hospital', the HAD may be used in community work. It is acceptable to and easily completed by the patient. It provides a distinction between the two concepts of depression and anxiety; the depression subscale is largely a reflection of anhedonia since this state seems to be the best clinical marker of the aspect of depression indicating disturbed neurobiological mechanisms (Snaith, 1992). The HAD has been translated into most European languages, also into Arabic, Israeli, Urdu, Japanese, and Chinese, and other translations are being considered.
Those wishing for information should send a stamped addressed envelope (A4 size) to the address below. A bibliography of studies validating the HAD in different settings will be included; in order to keep this up-to-date I should be pleased to receive reprints of all studies incorporating the use of the HAD.

SNaIth, R. P. (1992) Anhedonia. British Medical Journal (in press).

Academic Unit of Psychiatry

R. P. SNAITH

Clinical Sciences Building

St James's University Hospital

Leeds LS9 7TF

\section{Fingerprints and statistics}

SIR: I read with interest the paper by Mellor on dermatoglyphics and schizophrenia (Journal, April 1992, 160, 467-472). The author remarks that many of the studies involving dermatoglyphics have yielded ambiguous results (p. 467).

Some of this ambiguity may be due to the incorrect use of the $\chi^{2}$ test, where it is assumed that all observations are independent. Mellor (1968) analysed fingerprint patterns in schizophrenics and normal individuals with the pattern on a finger as the unit of observation (hence 500 individuals yield 5000 observations). However, pattern types on the fingers are not statistically independent. From Table 3 of Dr Mellor's recent paper it can be seen that around $80 \%$ of homologous fingers have the same pattern type!

Donner \& Donald (1988) have proposed an adjusted $\chi^{2}$ statistic which can be used when each individual provides several associated observations. They show that the results obtained from an analysis which ignores the dependency between observations are likely to be more statistically significant than those obtained from an analysis which takes this dependence into account. Thus statistically significant findings from the analysis of fingerprint patterns as independent observations may be misleading.

DONner, A. \& Donald, A. (1988) The statistical analysis of multiple binary measurements. Journal of Clinical Epidemiology, 41, 899-905.

Mellor, C. S. (1968) Dermatoglyphics in schizophrenia. Part I: Qualitative aspects. Part II: Quantitative study. British Journal of Psychiatry, 114, 1387-1397.

\author{
Department of Public Health Medicine \\ UMDS (Guy's Campus) \\ London SEI 9RT
}

